\title{
Actividad y Fuerza de Maseteros y Temporales de Pa- cientes Rehabilitados con Sobredentadura vs Pacientes dentados
}

\author{
Masseter and Temporalis Muscles Activity and Strength in Rehabilitated Patients \\ with Overdentures Versus Dentate Patients
}

Juan Alberto Aristizabal Hoyos”; Olga Patricia López Soto² \& Lina María López Soto

\begin{abstract}
ARISTIZABAL, H. J. A.; LÓPEZ, S. O. P. \& LÓPEZ, S. L. M. Actividad y fuerza de maseteros y temporales de pacientes rehabilitados con sobredentadura vs pacientes dentados. Int. J. Odontostomat., 11(2):224-230, 2017.

RESUMEN: La investigación de los músculos de la masticación, ayuda a un mejor entendimiento de la función oral al permitir un análisis cuantitativo de su actividad y fuerza. Comparar la actividad y fuerza de los músculos maseteros (M) y temporales (T) derechos (D) e izquierdos (I) de pacientes rehabilitados con sobre-dentaduras inferiores (RSI), con la de pacientes dentados con oclusión funcional (PD). Estudio de corte transversal , con muestreo intencional de 28 adultos RSI y 28 adultos PD .Se analizó la actividad muscular de M y T durante la masticación y contracción voluntaria máxima (CMV) mediante electromiografía de superficie; y la fuerza, mediante mioescanografía. La comparación de promedios se realizó mediante la prueba U de Mann Whitney. Los promedios de la CVM en el grupo ISD en micro-voltios (mV) fue: MD: 418,93 MI: 456,26 ; TD: 439,47 mV; TI: 505,54 mV; actividad en masticación: 198, $56 \mathrm{mV}$; la fuerza en libras fue: MD: 0,38 lb; Ml: 0,37lb. En el grupo control los promedios de la CVM en mV: MD: 829,45; MI: 793,53 ; TD: 830,76; TI: 887,82 ; Masticación MD: 172,79; Ml: 182,89; fuerza en lb : MD: o,53lb; MI:0,53 .La diferencia fue estadísticamente significativa en la fuerza, la CVM de M y T y en la masticación en el T y M derechos $(p<0.05)$. Hay diferencias en la fuerza y la actividad durante contracción voluntaria máxima y la masticación en maseteros y temporales de pacientes PRSI comparados con pacientes PD.
\end{abstract}

PALABRAS CLAVE: Músculo temporal; músculo masetero, actividad motora, fuerza de mordida (MeSH)

\section{INTRODUCCIÓN}

La masticación es una función compleja que incluye reflejos voluntarios y automáticos controlados por patrones generados por el sistema nervioso central y regulados por varios receptores (extero-propioreceptores y viscero-receptores) (Rilo et al., 2007). La pérdida de un diente natural puede rehabilitarse con prótesis soportadas por los dientes remanentes, por la mucosa alveolar o por medio de implantes oseo-integrados. Se ha sugerido que la evaluación funcional de una rehabilitación prostodóntica debe incluir la medida de la fuerza al morder y la valoración de los movimientos masticatorios y los registros electromiográficos de superficie de los músculos que intervienen en la masticación (Gartner et al., 2000; Ferrario et al., 2004; Feine \& Lund, 2006; Van Steenberghe \& Jacobs, 2006).

Las personas edéntulas, aún con prótesis totales bien adaptadas, tienen reducidas varias funciones orales, como la fuerza de mordida, la eficiencia masticatoria, los umbra- les táctiles y la percepción oral de la dureza y el espesor de los objetos (Manly et al., 1952; Karkazis, 2002). El patrón de masticación y la función masticatoria son diferentes en los portadores de prótesis dentales completas comparados con pacientes dentados (Jemt, 1981; Witter et al., 2013). La fuerza máxima de mordida en pacientes con sobre-dentaduras soportadas por implantes es 60 a 200 veces más alta que en pacientes con sobre-dentaduras convencionales (Van Kampen et al., 2002; Uçankale et al., 2012).

El examen clínico sin ayudas diagnósticas puede llevar a errores graves, este examen puede ser optimizado a través del uso de técnicas que midan objetivamente la función del Sistema Estomatognático (SEG), y que por lo tanto reduzcan el sesgo del diagnóstico estrictamente clínico. La investigación cuantitativa de los músculos que intervienen en las funciones del SEG, puede proporcionar conocimientos acerca de los mecanismos normales de su acción inte-

\footnotetext{
${ }^{1}$ Rehabilitador Oral, Profesor Asociado de la Universidad Autónoma de Manizales, Colombia.

2 Odontóloga, Profesora Asociada de la Universidad Autónoma, Colombia.

${ }^{3}$ Fonoaudiólga, Profesora Instructora de la Universidad Autónoma de Manizales, Colombia.
} 
grada y de su control nervioso. En la clínica, la investigación de los músculos de la masticación, puede ayudar a un mejor entendimiento de los problemas del paciente al permitir un monitoreo cuantitativo de los efectos del tratamiento (Tecco et al., 2007).

La electromiografía de superficie (EMGs) es considerada válida para la evaluación de la función muscular masticatoria (Mioche et al., 2004; Paphangkorakit et al., 2008), al permitir el registro de las corrientes eléctricas generadas por el músculo durante su actividad funcional. La EMGs se define como el conjunto de técnicas y procedimientos electrofisiológicos que registran la actividad eléctrica muscular producida espontáneamente o durante la contracción voluntaria como resultado de las variaciones de voltaje que se producen en las fibras musculares, como expresión de la despolarización de las membranas celulares durante la contracción (Kimura, 1989).

A nivel clínico la EMGs busca obtener datos de la amplitud de la actividad muscular en particular, índices de simetría entre dos músculos o grupos de músculos con diferentes componentes como la movilidad o el torque, también proporciona datos del dominio de la frecuencia y posibilita la realización de hipótesis acerca del contenido de las fibras de los músculos que lo componen (Farella et al., 2002; Kumar et al., 2003). Regularmente la EMGs es usada para realizar registros objetivos de la función y disfunción muscular (Ferrario et al., 2007; Castroflorio et al., 2008; De Felício et al., 2009).

Observaciones electromiográficas, en pacientes desdentados usuarios de prótesis dentales completas convencionales, revelan que la potencia de la actividad funcional de los músculos temporal anterior y masetero está significativamente reducida, en comparación con individuos que tienen dentición natural (Ingervall \& Hedegård, 1980; Tallgren et al., 1980; Tallgren et al., 1992; Chen et al., 2002; Naert et al., 2004). Esas alteraciones indican un compromiso de la capacidad funcional masticatoria.

Datos de investigaciones clínicas (Christensen \& Kundinger, 1991) sobre los patrones de los músculos maseteros y temporales, durante la función normal, en sujetos saludables sin alteraciones musculares explican que el masetero es el que más contribuye a las fuerzas isométricas que se hacen durante el apretamiento máximo (McCarroll et al., 1989; Visser et al., 1992), mientras que el temporal, que es un músculo posicionador, controla los movimientos mandibulares en funciones excursivas (Ahlgren, 1986; Nakamura et al., 1989; Kerstein, 2004).

Heckman et al. (2009) publicaron en el 2009 una investigación a 10 años donde mostraron la adaptabilidad funcional del sistema neuromuscular en pacientes edéntulos que habían sido registrados en las 4 diferentes etapas de rehabilitación oral: (1) Con prótesis totales viejas e insuficientes, (2) nuevas dentaduras completas, (3) sobre-dentaduras implanto-soportadas con 10 años de uso. Hubo cam- bios significativos entre los estados 2 y 3 que indicaron que la estabilización de las dentaduras a través de los implantes está acompañada de un incremento inmediato de los parámetros neuromusculares.

Este estudio pretende comparar la actividad y la fuerza de los músculos maseteros y temporales de un grupo pacientes rehabilitados con sobre-dentaduras implanto-soportadas con un grupo de pacientes dentados con oclusión funcional, utilizando mioescanografia y electromiografía de superficie.

\section{MATERIAL Y MÉTODO}

Este estudio de corte transversal realizó un muestreo por conveniencia que incluyó dos grupos: uno de 28 adultos desdentados totales inferiores rehabilitados con sobredentaduras colocadas sobre dos implantes no ferulizados con pilares tipo bola con un año de función en boca que asistían a las clínicas odontológicas de una entidad universitaria y el otro de 28 pacientes control dentados y con oclusión estable.

Como criterios de inclusión se consideraron un grupo de pacientes desdentados totales inferiores, que habían recibido dos implantes interforaminales rehabilitados con pilares tipo bola para soportar una sobredentadura inferior. Para el grupo de pacientes dentados se consideró que tuviesen más de 10 pares de dientes naturales en oclusión estable, sin rehabilitación con implantes. Los criterios de exclusión fueron haber recibido intervención en las sobredentaduras o en los implantes en una entidad externa a la que realizó el procedimiento original. La investigación fue aprobada por el Comité de Bioética (acta 16 de 2013). Se solicitó consentimiento informado a todos los pacientes según la norma 8430 del Ministerio de Salud de Colombia.

Se realizó el análisis de electromomiografía de superficie en los músculos maseteros y temporales durante la masticación y de la contracción voluntaria máxima utilizando un equipo de software Cadwe $\| \circledast$. Se tomaron los registros de potenciales de unidades motoras (MUP siglas en inglés) en cada caso. La fuerza muscular se determinó utilizando un mioescáner y los resultados se analizaron según los estándares guía.

El registro EMGs se realizó con el electrofisiógrafo y software Sierra ${ }^{\circledR}$ Wave ${ }^{\circledR}$ de Cadwell $囚$. Los parámetros de adquisición fueron: frecuencia de muestreo de $76.8 \mathrm{kHz}$, filtro pasa bandas de $10 \mathrm{a} 10 \mathrm{k} \mathrm{Hz}, 200 \mu \mathrm{V}$ de ganancia. Para la adquisición y almacenamiento de los registros se seleccionó el protocolo EMG. Se utilizaron electrodos de disco Cadwell $302139-200$ de acero inoxidable y 10 mm de diámetro.

Para la toma del registro de EMGs, los pacientes se ubicaron en una silla con espaldar, la espalda recta, brazos 
relajados, pies apoyados en el suelo, ojos abiertos y con la vista fija en una de las paredes de la sala. Previo al posicionamiento de los electrodos se limpió el rostro del paciente con algodón y etanol al $95 \%$, se utilizó el gel conductor Cadwell $202153-000$ con el fin de disminuir la aparición de artefactos debido a la baja conductancia. El electrodo de tierra, común a todos los demás pares de electrodos, se ubicó $2 \mathrm{~cm}$ por encima del nasion.

En este estudio se evaluaron la actividad muscular de los maseteros y los temporales. Se seleccionó una configuración monopolar para los electrodos de superficie, lo anterior puesto que esta configuración es más sensible a los cambios de la fuerza ejercida por un músculo. La posición de los electrodos se realizó así: Maseteros: se le solicitó al paciente apretar los dientes con fuerza mientras se realizó una palpación del músculo masetero, el electrodo activo se posicionó en la prominencia formada por este músculo al realizar la maniobra mencionada; el electrodo de referencia se ubicó $1 \mathrm{~cm}$ por debajo del lóbulo de la oreja. Temporales: se le solicitó al paciente apretar los dientes con fuerza mientras se realizaba una palpación del haz anterior del músculo temporal; el electrodo activo se posicionó en la prominencia formada por este músculo al realizar la maniobra mencionada, el electrodo de referencia se ubicó $1 \mathrm{~cm}$ por debajo.

Cada músculo se evaluó de manera independiente. En primer lugar se determinó la contracción voluntaria máxima (CVM) de los músculos maseteros (10 segundos de oclusión máxima) y temporales (10 segundos oclusión máxima). A continuación, cada paciente masticó un chicle hasta que se obtuvo registro del patrón de masticación. Para este estudio, se analizaron las MUP registradas para cada uno de los músculos y en cada una de las maniobras. Para las medidas de contracción voluntaria máxima se registró la medida de amplitud máxima de la contracción, como la media de voltaje pico a pico de 4 ondas dentro del registro.

La fuerza de los músculos temporales y maseteros fue tomada mediante mioescanografía. El mioescaner utilizado fue un equipo Pounds Myoescanner, NeilcoTechnologyinc, D-926119T8 Hoxt, 1 volt=F.S, pt 2500. La fuerza o tensión fisiológica de los músculos, fue censada por un plug, que permitió medir la fuerza de contracción de maseteros y temporales. Para la realización del examen se solicitó al paciente sentarse cómodamente con la cabeza apoyada sobre la pared, y los pies apoyados sobre el piso. Primero se evaluó la contracción del músculo masetero derecho, se solicitó al paciente apretar los dientes en cierre, el examinador palpó con las yemas de los dedos índice y medio el músculo repitiendo tres veces la misma acción con el fin de determinar su localización. Luego se dio inicio al examen con la calibración del mioescaner, de modo que quedara exactamente localizado sobre la medida 0.0 libras, se dispuso la platina sobre el masetero y se solicitó nuevamente al paciente una oclusión máxima; se realizó el mismo procedimiento en el masetero izquierdo.
Para evaluar la fuerza del temporal derecho se solicitó al paciente apretar los dientes, el examinador palpó con las yemas de los dedos índice y medio el vientre anterior del músculo repitiendo tres veces la misma acción con el fin de determinar su localización. Luego se dio inicio al examen con la calibración del mioescaner, de modo que quede exactamente localizado sobre la medida 0.0 libras, se dispuso la platina sobre el vientre anterior del temporal y se solicitó nuevamente al paciente apretar los dientes en cierre posterior; se realizó el mismo procedimiento en el temporal izquierdo. Todas las medidas de mioescanografía se repitieron tres veces y se registró el valor más alto alcanzado en cada medida.

El análisis estadístico se realizó utilizando el software Statistical Package for the Social Sciences (SPSS $®$ IBM $®$ v.20). Las comparaciones se realizaron con la prueba $U$ de Mann-Withney debido a que la mayoría de variables no cumplieron con las pruebas de normalidad (Kormogorov-Smirnov) ni homocedasticidad (test de Levene); estas variables se analizaron bajo un intervalo de confianza del $95 \%$.

\section{RESULTADOS}

El grupo de pacientes dentados mostró mayor actividad muscular en CVM de los músculos maseteros y temporales que los pacientes rehabilitados con sobre-dentadura. Esta diferencia fue estadísticamente significativa ( $p>0.05)$. La actividad de maseteros y temporales durante la masticación fue similar en los dos grupos (Tabla I).

Resultados de la mioescanografía. Los pacientes dentados presentaron mayor fuerza de contracción máxima del músculo orbicular, contracción de los maseteros derecho e izquierdo y mayor fuerza de extensión máxima lingual que los pacientes rehabilitados con sobredentadura, la diferencia entre los grupos fue estadísticamente significativa. $(p<0.05)$ (Tabla II).

\section{DISCUSIÓN}

En esta investigación se analizaron 28 pacientes con prótesis total superior y sobre-dentadura mandibular soportadas sobre dos implantes no ferulizados con ajustes tipo bola y se compararon con 28 sujetos que presentan dentición natural. Todos los pacientes rehabilitados con sobredentadura se encontraban satisfechos con sus prótesis y reportaban una adecuada masticación, llevaban usándolas un año tiempo mayor al considerado suficiente para el desarrollo de una buena actividad y fuerza muscular (Gartner et al.).

En este estudio, durante la CMV, los pacientes que usaban sobre-dentaduras registraron menor actividad electromiográfica que aquellos pacientes con dentición 
Tabla I. Diferencia estadística de los promedios de la amplitud de la actividad medida en $\mathrm{mV}$, de los maseteros y temporales en contracción voluntaria máxima (CMV) y masticación, en pacientes con sobredentadura y dentados.

\begin{tabular}{|c|c|c|c|}
\hline Músculos & Grupos & Media & $\begin{array}{c}\text { Diferencia estadística } \\
\text { p valor }\end{array}$ \\
\hline \multicolumn{4}{|c|}{ Masetero Izquierdo } \\
\hline \multirow[t]{2}{*}{ CVM } & Sobre-dentadura & & \multirow[t]{2}{*}{$0,000^{*}$} \\
\hline & Dentados & 793,53 & \\
\hline \multirow[t]{2}{*}{ Masticación } & Sobre-dentadura & 164,96 & \multirow[t]{2}{*}{0,184} \\
\hline & Dentados & 182,89 & \\
\hline \multicolumn{4}{|c|}{ Masetero Derecho } \\
\hline & Sobre-dentadura & $\begin{array}{l}418,93 \\
82945\end{array}$ & \multirow{3}{*}{$0,000^{*}$} \\
\hline CVM & Dentados & & \\
\hline \multirow[t]{2}{*}{ Masticación } & Sobre-dentadura & 172,48 & \\
\hline & Dentados & 172,79 & 0,972 \\
\hline \multicolumn{4}{|c|}{ Temporal Derecho } \\
\hline \multirow[t]{2}{*}{ CVM } & Sobre-dentadura & 439,47 & \multirow[t]{2}{*}{$0,000^{*}$} \\
\hline & Dentados & 830,76 & \\
\hline \multirow[t]{2}{*}{ Masticación } & Sobre-dentadura & 180,32 & \multirow[t]{2}{*}{$0,052^{*}$} \\
\hline & Dentados & 208,14 & \\
\hline \multicolumn{4}{|c|}{ Temporal Izquierdo } \\
\hline \multirow[t]{2}{*}{ CVM } & Sobre-dentadura & 505,54 & \multirow[t]{2}{*}{$0,000^{*}$} \\
\hline & Dentados & 887,82 & \\
\hline \multirow[t]{2}{*}{ Masticación } & Sobre-dentadura & 198,56 & \\
\hline & Dentados & 198,64 & 0,844 \\
\hline
\end{tabular}

${ }^{*}$ Estadísticamente significativo.

\begin{tabular}{lllc}
\hline \multicolumn{1}{c}{ Músculos } & \multicolumn{1}{c}{ Grupos } & Promedio & $\begin{array}{c}\text { Diferencia estadística } \\
\text { p valor }\end{array}$ \\
\hline Fuerza contracción máxima orbicular & Sobredentadura & 0,43 & $0,035^{*}$ \\
& Dentados & 0,53 & \\
Fuerza contracción masetero derecho & Sobredentadura & 0,38 & $0,0001^{*}$ \\
& Dentados & 0,53 & \\
Fuerza contracción masetero izquierdo & Sobredentadura & 0,37 & $0,0001^{*}$ \\
& Dentados & 0,53 & \\
\hline
\end{tabular}

natural. Esos resultados son similares a los hallados por Fontijn-Tekamp et al. (2000) quienes registraron una fuerza de mordida con sobre-dentadura mayor a las prótesis totales pero inferior a la dentición natural. Los implantes que retienen sobre-dentaduras son una opción eficaz para proporcionar una mejor función masticatoria en los pacientes edéntulos (Naert et al., 1999). Desde la perspectiva neurofisiológica, los implantes retenidos con sobre-dentaduras trabajan en ausencia de los mecano-receptores del ligamento periodontal. Considerando las funciones de estos mecano-receptores, el resultado es una alteración de las capacidades de la percepción oral, en especial el reflejo inhibitorio (Horio \& Kawamura, 1989), por lo que es común que los pacientes que usan sobre-dentaduras presenten una actividad muscular mayor durante la masticación, inclusive que en personas dentadas (Karkazis, 2002). Esto no coincidió con los resultados de esta investigación, donde los pacientes registraron valores de actividad muscular menores a los pacientes dentados. Esto podría explicarse porque durante el periodo edéntulo podría ocurrir una atrofia muscular que disminuye la actividad electromiográfica, un lapso prolongado de tiempo sin tener una rehabilitación adecuada de la función masticatoria, puede hacer que la restauración de la actividad muscular se dé progresivamente y no se 
refleje en los primeros años en los valores electromiográficos (Ferrario et al.). Los individuos de la presente investigación estuvieron durante más de 10 años con una rehabilitación de prótesis total mucosoportada.

Diferente a éste trabajo, Tartaglia et al. (2008) concluyeron que electromiográficamente durante el apretamiento los pacientes con prótesis son funcionalmente equivalentes a los pacientes con dentición natural pero que los pacientes dentados durante la masticación tuvieron una mayor coordinación muscular que los pacientes con implantes oseointegrados. La coordinación neuromuscular durante la masticación (patrones alternados de contracción e inhibición) parecen depender de la clase de soporte de la superficie oclusal. En general, los pacientes con rehabilitación sobre implantes parecen usar un mayor número de músculos que los pacientes dentados, con un incremento de los patrones de oclusión bilateral comparado con la masticación unilateral, más frecuente, de los individuos dentados.

Otra explicación para haber obtenido una actividad y una fuerza muscular menor en los pacientes con sobre-dentadura respecto a los pacientes dentados, es el hecho de que la función táctil es obviamente diferente que la de los dientes naturales. La ausencia del ligamento periodontal debe ser compensada por la función exteroceptiva de los receptores de la encía, de la mucosa alveolar y del hueso (Jacobs \& van Steenberghe, 1993). También, se ha sugerido que la prótesis colocada sobre implantes pueden activar propioceptores remotos a través de la transmisión de vibraciones vía los huesos faciales. Aún más, se han encontrado cambios en la inervación de la mucosa oral después de la rehabilitación con sobredentaduras (Garzino et al., 1996). Todas están compensaciones, pueden no ser suficientes para reflejar una actividad y una fuerza muscular similar a la del paciente dentado.

Los pacientes que reciben sobre-dentaduras disminuyen la actividad electromiográfica de los músculos maseteros y temporales, pero aumentan la simetría de la actividad del masetero e inhibien la postura de la dinámica mandibular, mientras mantienen el ciclo similar de masticación que tenía con la prótesis anterior (Piancino et al., 2005). Cuando existe una estabilidad protésica, como en el caso de las sobre-dentaturas, el músculo temporal no está en hiperactividad, permitiendo a la musculatura permanecer en balance y mantener la postura y la posición de reposo (Santos et al., 2008).

En un artículo de revisión de literatura, publicado por Dellavia et al. (2014), los estudios seleccionados indicaban que los pacientes rehabilitados con dentaduras fijas sobre implantes dentales comparados con sujetos dentados tenían un equilibrio muscular satisfactorio en actividades estáticas, pero con algunas dificultades durante la masticación. Desde el punto de vista neurofisiológico las sobre-dentaduras implanto-retenidas al no presentar mecano receptores por la falta de ligamento periodontal dan como resultado una considerable alteración de las habilidades de percepción sensorial oral. Sin embargo la activación de mecano receptores periostales, o terminaciones nerviosas intraóseas pueden ser una explicación para la capacidad de la mayoría de los pacientes con implantes para discriminar espesores interoclusales y percibir cargas (Karkazis, 2002). El soporte de la mucosa ofrece una entrada neural adicional a través de la estimulación de extero-receptores mucosales los cuales parecen asumir el control, en cierta medida, del papel de los receptores periodontales perdidos (Karkazis \& Kossioni, 1998).

El estudio de Uram-Tuculescu et al. (2015) encontró una actividad muscular masticatoria relativa 2.57 veces más alta para los usuarios de dentaduras que para los sujetos dentados $(p<0001)$ mientras que la duración de la secuencia de masticación incrementó con la fuerza de fractura de los alimentos. Esto no difiere significativamente respecto a los pacientes dentados y concluyen que los aumentos observados en la actividad muscular masticatoria relativa para los usuarios de dentaduras comparados con los sujetos dentados durante el procesamiento oral de los alimentos probablemente refleja los esfuerzos mecánicos suplementarios para acomodar el uso de prótesis dentales para la preparación del bolo alimenticio antes de la deglución (UramTuculescu et al.).

Entre las limitaciones de este estudio está el hecho de haber considerado una muestra por conveniencia: los pacientes no fueron seleccionados aleatoriamente, por lo que la extrapolación de los resultados a una población más amplia no es posible ; tampoco se realizó en esta investigación un estudio de la eficacia del acto masticatorio que pudo haber explicado mejor el comportamiento muscular.

Los tópicos acerca del tratamiento de pacientes edéntulos más investigados por la rehabilitación oral incluyen la función de los músculos masticatorios, la oclusión dental, las relaciones craneofaciales y la articulación temporomandibular . Esto indica la importancia de considerar la electromiografía de superficie con el fin de hacer posible que este examen se realice en forma rutinaria para el diagnóstico y el seguimiento de los tratamientos rehabilitadores.

\section{CONCLUSIONES}

1.- El grupo de pacientes dentados mostró mayor actividad muscular en CVM de los músculos maseteros y temporales que los pacientes rehabilitados con sobre-dentadura $(p<0.05)$.

2.- Los pacientes dentados presentaron mayor fuerza de contracción máxima del músculo orbicular, contracción de los maseteros derecho e izquierdo y mayor fuerza de extensión máxima lingual que los pacientes rehabilitados con sobredentadura $(p<0.05)$. 
Para optimizar la comparación de la actividad de los músculo masticatorios entre pacientes dentados y los que reciben sobre-dentaduras, sería importante medir la actividad electromiográfica antes de la cirugía y de la colocación de los implantes. Además, la actividad de todos los músculos masticatorios debería ser medida simultáneamente en el lado de trabajo y balanza para determinar la actividad de cada uno en el patrón masticatorio.

ARISTIZABAL, H. J. A.; LÓPEZ, S. O. P. \& LÓPEZ, S. L. M. Masseter and temporalis muscles activity and strength in rehabilitated patients with overdentures versus dentate Patients. Int. J. Odontostomat., 11(2):224-230, 2017.

ABSTRACT: Mastication muscles research help a better understanding of the patient's oral function by allowing quantitative monitoring of their activity and strength. To compare the masseter (M) and the right (RT) and left (LT) temporalis muscles activity and strength in rehabilitated patients with implant overdentures with functional occlusion dentate patients. Cross sectional study, that examined a convenience sample of 28 edentulous lower jaw adults (group) who had undergone an overdenture rehabilitation treatment on two unsplinted implants and 28 dentate adults with stable occlusion (control group). $\mathrm{M}$ and $\mathrm{T}$ muscle activity was analyzed during mastication. A surface electromyography served to analyze maximum voluntary contraction (MVC) and a mioescanography served to analyze strength. The MannWhitney $U$ test was used to compare average results. The MVC average results of the ISD group in microvolts $(\mathrm{mV})$ were RM: 418.93, LM: 456.26; RT: $439.47 \mathrm{mV}$; LT: 505.54 $\mathrm{mV}$; mastication activity: $198.56 \mathrm{mV}$; strength in pounds was RM: 0.38 lbs; LM: 0.37 lbs. The control group showed MVC average results in mV: RM: 829.45; LM: 793.53; RT: 830.76; LT: 887,82; mastication RM: 172.79; LM: 182, 89; strength in pounds: RM: $0.53 \mathrm{lbs}$; LM: 0,53 lbs. Strength exhibited a statistically significant difference of MVC in M and T muscles; and mastication in RT and RM $(p<0.05)$. There are strength and activity muscle differences during maximum voluntary contraction (MVC) and mastication on both masseter and temporalis muscles of rehabilitated patients with implant overdentures compared to dentate patients.

KEY WORDS: Temporal muscle; Masseter muscle; Motor activity; Bite force (MESH)

\section{REFERENCIAS BIBLIOGRÁFICAS}

Ahlgren, J. EMG pattern of temporalis in normal occlusion. Eur. J. Orthod., 8(3):185-91, 1986.

Castroflorio, T.; Bracco, P. \& Farina, D. Surface electromyography in the assessment of jaw elevator muscles. J. Oral Rehabil., 35(8):638-45, 2008.

Chen, L.; Xie, Q.; Feng, H.; Lin, Y. \& Li, J. The masticatory efficiency of mandibular implant-supported overdentures as compared with tooth-supported overdentures and complete dentures. J. Oral
Implantol., 28(5):238-43, 2002.

Christensen, L. V. \& Kundinger, K. K. Activity index and isometric contraction velocity of human jaw muscles. J. Oral Rehabil., 18(6):555-61, 1991

De Felício, C. M.; Sidequersky, F. V.; Tartaglia, G. M. \& Sforza, C. Electromyographic standardized indices in healthy Brazilian young adults and data reproducibility. J. Oral Rehabil., 36(8):57783, 2009

Dellavia, C.; Rosati, R.; Del Fabbro, M. \& Pellegrini, G. Functional jaw muscle assessment in patients with a full fixed prosthesis on a limited number of implants: a review of the literature. Eur. J. Oral Implantol., 7 Suppl. 2:S155-69, 2014.

Farella, M.; Van Eijden, T.; Baccini, M. \& Michelotti, A. Task-related electromyographic spectral changes in the human masseter and temporalis muscles. Eur. J. Oral Sci., 110(1):8-12, 2002.

Feine, J. S. \& Lund, J. P. Measuring chewing ability in randomized controlled trials with edentulous populations wearing implant prostheses. J. Oral Rehabil., 33(4):301-8, 2006.

Ferrario, V. F.; Tartaglia, G. M.; Luraghi, F. E. \& Sforza, C. The use of surface electromyography as a tool in differentiating temporomandibular disorders from neck disorders. Man. Ther., 12(4):372-9, 2007.

Ferrario, V. F.; Tartaglia, G. M.; Maglione, M.; Simion, M. \& Sforza, C. Neuromuscular coordination of masticatory muscles in subjects with two types of implant-supported prostheses. Clin. Oral Implants Res., 15(2):219-25, 2004.

Fontijn-Tekamp, F. A.; Slagter, A. P.; Van Der Bilt, A.; Van 'T Hof, M. A.; Witter, D. J.; Kalk, W. \& Jansen, J. A. Biting and chewing in overdentures, full dentures, and natural dentitions. J. Dent. Res., 79(7):1519-24, 2000.

Gartner, J. L.; Mushimoto, K.; Weber, H. P. \& Nishimura, I. Effect of osseointegrated implants on the coordination of masticatory muscles: a pilot study. J. Prosthet. Dent., 84(2):185-93, 2000.

Garzino, M.; Ramieri, G.; Panzica, G. \& Preti, G. Changes in the density of protein gene product 9.5-immunoreactive nerve fibres in human oral mucosa under implant-retained overdentures. Arch. Oral Biol., 41(11):1073-9, 1996.

Heckmann, S. M.; Heussinger, S.; Linke, J. J.; Graef, F. \& Pröschel, P. Improvement and long-term stability of neuromuscular adaptation in implant-supported overdentures. Clin. Oral Implants Res., 20(11):1200-5, 2009.

Horio, T. \& Kawamura, Y. Effects of texture of food on chewing patterns in the human subject. J. Oral Rehabil., 16(2):177-83, 1989.

Ingervall, B. \& Hedegård, B. An electromyographic study of masticatory and lip muscle function in patients with complete dentures. J. Prosthet. Dent., 43(3):266-71, 1980.

Jacobs, R. \& van Steenberghe, D. Comparison between implantsupported prostheses and teeth regarding passive threshold level. Int. J. Oral Maxillofac. Implants, 8(5):549-54, 1993.

Jemt, T. Chewing patterns in dentate and complete denture wearers - recorded by light-emitting diodes. Swed. Dent. J., 5(5-6):199205, 1981.

Karkazis, H. C. \& Kossioni, A. E. Surface EMG activity of the masseter muscle in denture wearers during chewing of hard and soft food. J. Oral Rehabil., 25(1):8-14, 1998.

Karkazis, H. C. EMG activity of the masseter muscle in implant supported overdenture wearers during chewing of hard and soft food. J. Oral Rehabil., 29(10):986-91, 2002.

Kerstein, R. B. Combining technologies: a computerized occlusal analysis system synchronized with a computerized electromyography system. Cranio, 22(2):96-109, 2004.

Kimura, J. Electrodiagnosis in Diseases of Nerve and Muscle: Principles and Practice. $2^{\text {nd }}$ ed. Philadelphia, F. A. Davis Company, 1989.

Kumar, S.; Narayan, Y. \& Amell, T. Power spectra of 
ARISTIZABAL, H. J. A.; LÓPEZ, S. O. P. \& LÓPEZ, S. L. M. Actividad y fuerza de Maseteros y Temporales de pacientes rehabilitados con sobredentadura vs pacientes dentados. Int. J. Odontostomat., 11(2):224-230, 2017.

sternocleidomastoids, splenius capitis, and upper trapezius in oblique exertions. Spine J., 3(5):339-50, 2003.

Manly, R. S.; Pfaffman, C.; Lathrop, D. D. \& Keyser, J. Oral sensory thresholds of persons with natural and artificial dentitions. J. Dent. Res., 31(3):305-12, 1952.

McCarroll, R. S.; Naeije, M. \& Hansson, T. L. Balance in masticatory muscle activity during natural chewing and submaximal clenching. J. Oral Rehabil., 16(5):441-6, 1989.

Mioche, L.; Bourdiol, P.; Monier, S.; Martin, J. F. \& Cormier, D. Changes in jaw muscles activity with age: effects on food bolus properties. Physiol. Behav., 82(4):621-7, 2004.

Naert, I.; Alsaadi, G. \& Quirynen, M. Prosthetic aspects and patient satisfaction with two-implant-retained mandibular overdentures: a 10-year randomized clinical study. Int. J. Prosthodont., 17(4):401-10, 2004.
Dirección para correspondencia: Juan Alberto Aristizabal Hoyos Universidad Autónoma de Manizales Antigua Estación del Ferrocarril Manizales COLOMBIA

E-mail:jaristi@autonoma.edu.co

Recibido :13-03-2017

Aceptado:05-06-2017 